\title{
Development of a simulation model for the spread of COVID-19 coronavirus infection in Kaluga region
}

\author{
Alexey Tkachenko ${ }^{* 1}$, Denis Lavrentev ${ }^{1}$, Maksim Denisenko ${ }^{1}$, and Valentina Kuznetsova ${ }^{2}$ \\ ${ }^{1}$ Kaluga State University named after K.E.Tsiolkovski, 26, Stepan Razin St., 248023, Kaluga, Russia \\ ${ }^{2}$ Financial University, Kaluga Branch, 17 Chizhevskogo 248016, St., Kaluga, Russia
}

\begin{abstract}
The development of the situation around Covid-19 is an urgent problem for the whole world, and it is still not known exactly how the pandemic will develop. However, based on the data of the virus spread to date, it is possible to model the approximate outcome of the activities of humanity in the fight against Covid-19. To develop this model, we used a simulation tool-AnyLogic (Personal Learning Edition) with support for the Java programming language. This software has a large analytical functionality. When creating the Covid-19 infection model, the free version of the program was used. This model is only relevant if the pandemic continues at the same pace. There are many factors that can deviate from the presented model in any direction: early release from restrictive measures, virus mutations, forced vaccination of the population, and other measures that directly affect the main indicators of infection development. In the process of modelling the spread of Covid-19 coronavirus infection in the Kaluga region, results were obtained that reflect the rate of development of the pandemic. Thus, it is possible to identify the weaknesses of the fight against viruses and further minimize the rate of development of the disease.
\end{abstract}

\section{Introduction}

Modeling is an integral part of the predictive function of science. The modeling method has found its application in many areas of human activity, including medicine.

The modeling method in medicine is a tool that allows you to establish the relationship between theory and practice. Most medical research cannot be done experimentally, but simulations can minimize the risk and achieve a successful outcome.

The development and spread of infectious diseases is an integral companion of the evolution of mankind. The quality of medical care is improving every year. However, it is impossible to predict where and what type of new disease will appear.

In 2020, the population of the entire world faced the coronavirus infection Covid-19. The unexpectedly high rate of growth in the number of infected people and the lack of experience in dealing with such a serious disease have led to large human losses: at the time of writing, the number of deaths from Covid-19 in the world exceeds three million people.

\footnotetext{
* Corresponding author: TkachenkoAL@tksu.ru
} 
The development of the situation around Covid-19 is an urgent problem for the whole world, and it is still unknown exactly how the pandemic will develop. However, based on the data on the spread of the virus to date, it is possible to model the approximate outcome of human activities in the fight against Covid-19. The model will also allow you to check the consequences of a specific action that medicine wants to take against a coronavirus infection without risking the lives of real people.

\section{Research methodology}

To develop this model, the technology of the AnyLogic software product was used. This software has a large analytical functionality that allows you to simulate completely different situations in the course of a person's activity. A large abundance of automatic calculations allows you to assemble a computer model that is as close to reality as possible. When creating the Covid-19 infection model, the free version of the program was used, this version restricts the analysis of the infection prediction result in some situations.

The main functionality of the Covid-19 distribution model is storage devices that dynamically store information about a specific parameter. In our scheme, 7 drives were used:

1. Potentially susceptible people-Susceptible

2. People who are already infected but can't infect others-Exposed

3. People who can spread the virus and infect other people - Infectious

4. People who are undergoing the treatment process-Cured

5. People who have recovered and acquired antibodies-Recovered

6. People who have successfully been vaccinated - Vaccinated

7. People who could not recover from the infection before the fatal outcome - Dead

The main parameters of this system are:

- Total population (TotalPopulation);

* Rating of contact between people in one day (ContactRateInfectious) - this parameter is calculated in the range from 0 to 1 ;

* Probability of transmission (Infection) - this parameter takes values from 0.1 to 0.2 [1];

* Incubation period (AverageIncubationTime) - lasts 11 days [2];

* The average duration of the disease (AverageIllnessDuration) is 25 days [3];

* Probability of death (DeathProbability) - takes values from 0 to 0.01 [4];

* The probability of recovery from infection WITHOUT antibody production is 0.2 ;

* The probability of successful vaccination (VaccineProbability) is 0.91[5];

* Vaccination rating among the population (Vaccination) - takes values from 0 to 0.1 .

As you can see, some parameters have several undefined values. These changes are present in connection with certain events in the life of the population (Fig. 1). The key events that directly affect the fight against the development of infection were selected:

1. Appearance of restrictions: mask mode, self-isolation (transition between stages -1 month) [6]

1.1. Stage before restrictions:

* Increased rating of contact between people: the population lives a normal life, various events are held regardless of the number of participants.

* Increased risk of infection: the population does not observe the rules of hygiene to the proper extent, the treatment of premises is carried out without additional sanitation. 


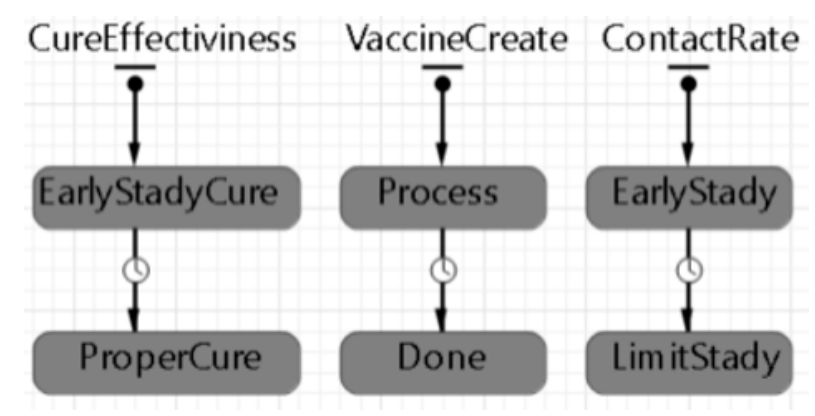

Fig. 1. Events that affect the spread of Covid-19 coronavirus infection.

1.2. Stage after restrictions (mask mode, self-isolation and other measures):

* The rating of contact between people has significantly decreased: almost all events have been canceled, the population observes home mode.

* The development of Covid-19 has slowed down: compliance with the mask regime, as well as maintaining a distance between people, significantly reduced the spread of infection.

2. Effectiveness of treatment of Covid-19 infection (transition between stages -2 months):

2.1. Early stage of treatment quality:

* The quality of infection treatment is unsuccessful: there is no complete information about the disease, medicine is developing an effective treatment for infection.

* The probability of death is increased: the population is not prepared for a pandemic, drugs only slow down the disease, but do not cure patients.

2.2. Settled stage:

* The quality of treatment has improved significantly: medicine has developed a more effective treatment for Covid-19.

* The probability of death decreased by several orders of magnitude: patients were given the necessary medications designed to maximize the chance of recovery.

3 . The process of vaccination of the population (transition between stages $8-9$ months):

3.1. Creating a vaccine:

* The vaccine is developed in laboratories: medicine collects the necessary data about the virus, experiments with drugs and the virus itself.

- No successful vaccination: the developed vaccines developed during this time period are not sufficiently successful.

3.2. The vaccine has been created:

* The vaccine is being delivered to different hospitals and care centers: the rate of development of the pandemic is slowing down again due to successful vaccination of the population.

* The probability of successful vaccination reaches $91 \%$.

The relationship between drives, parameters, and events is formed by threads that transfer a certain amount of data between drives (Fig. 2):

- The rate of transmission of the stream from the drive susceptible to infection to the drive of the latent stage of infection (ExposedRate) was calculated as follows: Infectious*ContactRateInfectious*Infection*Susceptible/TotalPopulation.

- The transfer rate of the stream from the latent stage of infection to the infected stage (InfectiousRate) was calculated as follows: Exposed/AverageIncubationTime.

- The rate of transmission of the stream from the drive of the infected to the drive of the dead (DeadRate) was calculated as follows: Infectious*DeathProbability/AverageIllnessDuration. 


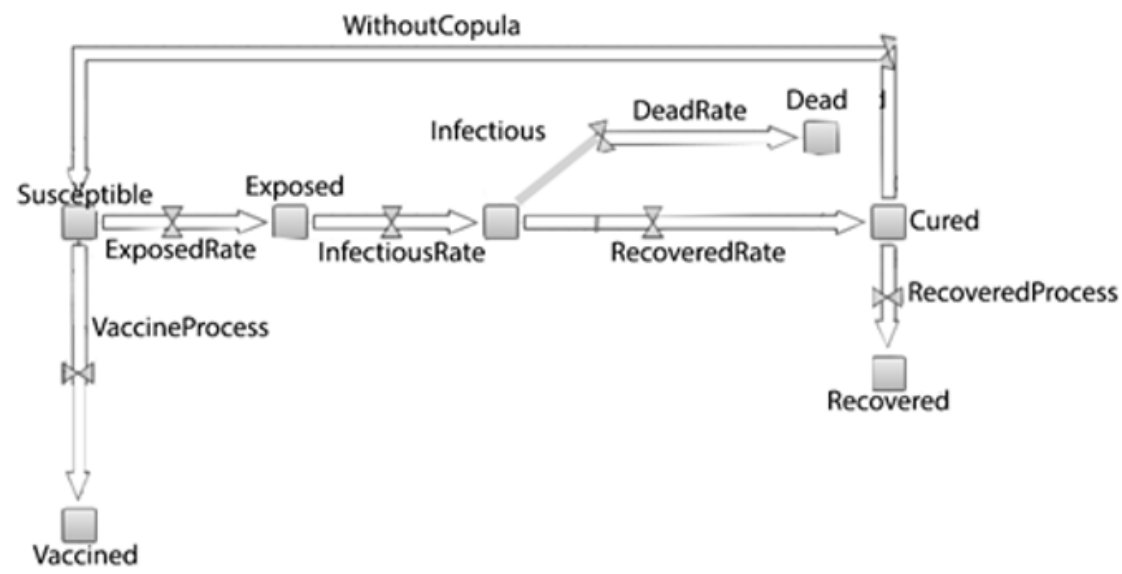

Fig. 2. Threads and storage devices.

- The rate of transfer of the flow from the drive of infected to the drive of those undergoing treatment was calculated as follows: Infectious*ProcessCure/AverageIllnessDuration.

- As a result of treatment, a certain percentage of patients who have been ill do not acquire antibodies, therefore, they may become infected again (go to the stage of Susceptible). The rate of transfer of the flow from the storage of those undergoing treatment to the storage of those susceptible to infection (WithoutCopula) was calculated as follows: Cured*WithoutCopulaProbability from the storage of those undergoing treatment to the storage of the fully cured (Recovered) is equal to the difference between Cured and WithoutCopula.

- The rate of transfer of the flow from the storage of susceptible to infection to the storage of vaccinated (Vaccinated) was calculated as follows: Susceptible*Vaccination*VaccineProbability.

As a visual representation of the model of the development of coronavirus infection, a time schedule was chosen that includes four key storage devices: Infectious, Recovered, Dead, Vaccinated (Fig. 3):

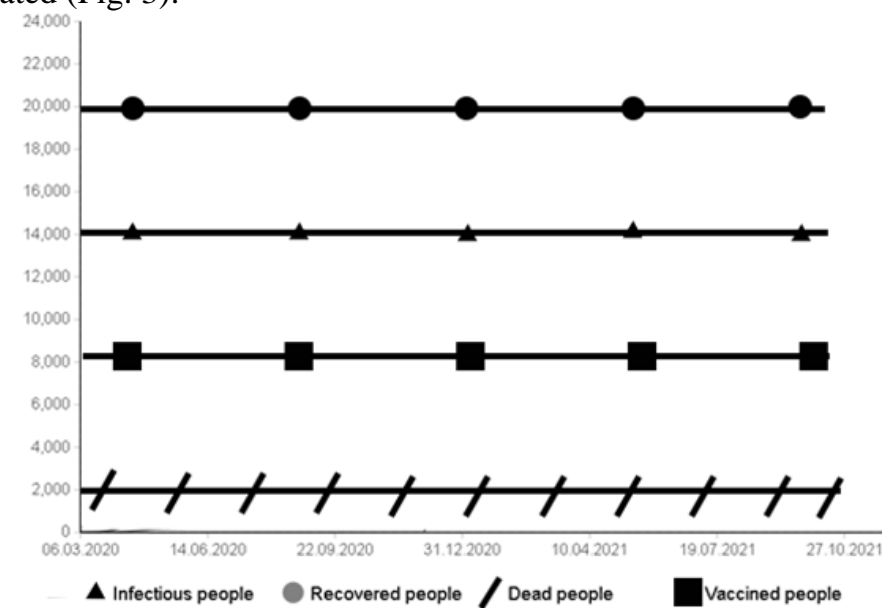

Fig. 3. Timeline of the spread of Covid-19 coronavirus infection.

1. Infectious people displays the data stored in the Infectious storage device.

2. Recovered people displays the sum of the data of the two drives: Recovered + Vaccinated. 
3. Dead people displays the data of the Dead drive.

4. Vaccinated people displays the data of the Vaccined drive.

\section{Results of the study}

I. The results of forecasting within the borders of one region (the Kaluga region was selected for the study).

The Kaluga region was chosen as an example for the study, since it is a region where the authors study and work, respectively, they could observe with their own eyes the development of infection and the introduction of restrictive measures imposed by the administrative authorities of the region. The Kaluga region is also located at a relatively short distance from Moscow and the Moscow region, where the disease situation was the most severe, which undoubtedly affected the growth rate of the number of infected people in the study region. In addition, the Kaluga State University named after K. E. Tsiolkovsky provided methodological assistance in the preparation of this article.

As a result of creating a model of the spread of Covid-19 coronavirus infection in the Kaluga region, the following conclusions were formulated (Fig. 4):



Fig. 4. Model of the spread of Covid-19 coronavirus infection in the Kaluga region. Time range 2 years from the beginning of infection during vaccination.

1. Subject to the implementation of restrictive measures to combat the pandemic and further vaccination of the population, the final victory over the new corona virus infection in the region will be achieved by 2024 .

2. The restrictive measures quickly introduced by the governor of the region significantly reduced the rate of infection of the population by reducing contacts between people during self-isolation, wearing masks and observing social distance.

3. In the absence of vaccination, the number of infected people will increase by $20 \%$ in 2 years from the beginning of infection. Victory over the virus is possible only with the development of antibodies and will come by 2028 . However, the virus will not completely disappear from the life of the population (see Fig. 5) 


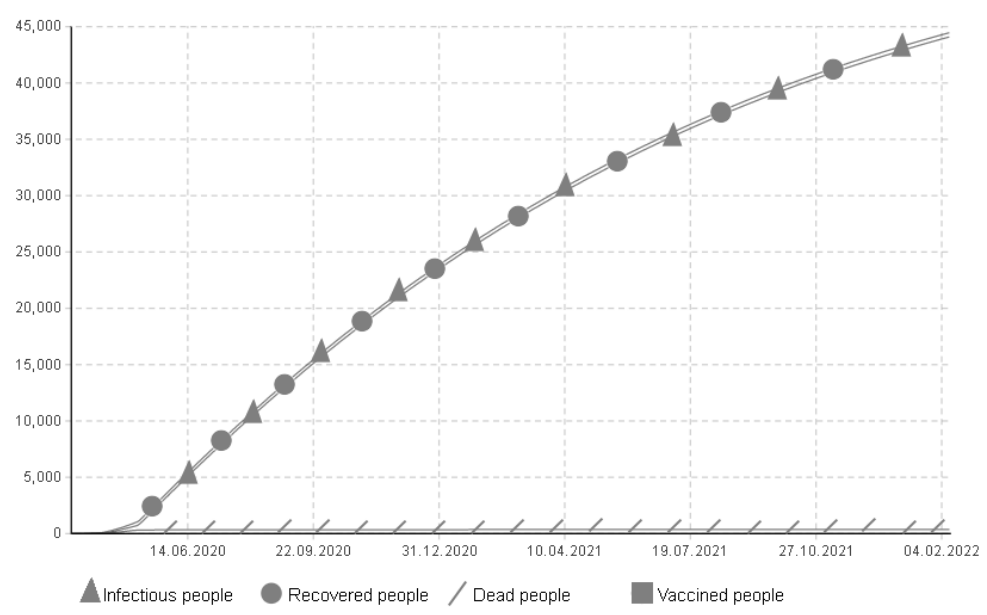

Fig. 5. Model of the spread of Covid-19 coronavirus infection in the Kaluga region. Time range 2 years from the beginning of infection without vaccination.

II. The results of forecasting within the borders of a single state (the United States of America was chosen for the study).

At the time of writing, the number of people affected by the infection is $10 \%$ of the total population of the state. Thus, the United States is the most striking example of the fight against this disease in the world, which is the most suitable material for research and modeling. If a correct and adequate prognosis is made, the competent actions of the authorities based on it can minimize the further spread of infection and reduce the risks of the disease for ordinary citizens.

As a result of the creation of a model for the spread of Covid-19 coronavirus infection in the United States of America, the following conclusions were formulated (Fig. 6):

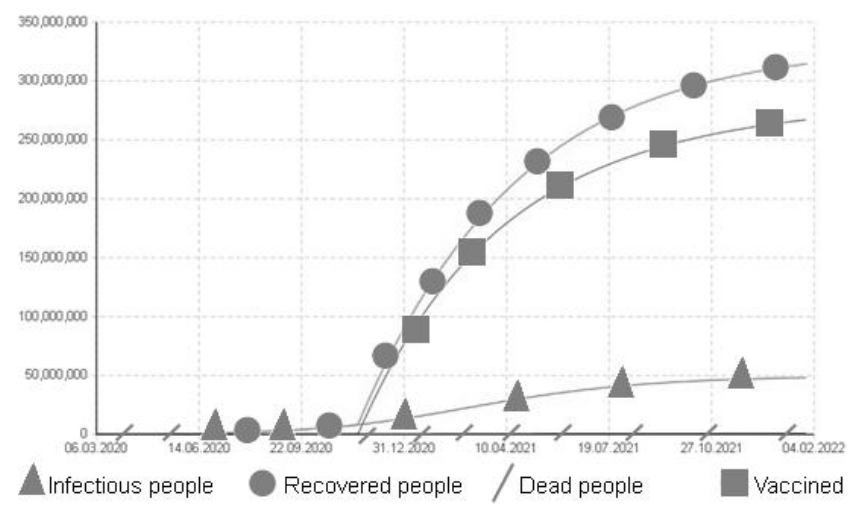

Fig. 6. Model of the spread of Covid-19 coronavirus infection in the United States of America. Time range 2 years from the beginning of infection during vaccination.

1. The turning point in the fight against coronavirus infection in the United States came after the development of the vaccine, which had a positive impact on the number of people with immunity to Covid-19. Also, vaccination has significantly slowed the rate of spread, thanks to the large number of people with developed antibodies against the virus. 
2. With the introduction of restrictive measures in the country, the number of confirmed cases of death has sharply decreased, and the quality of treatment has improved, which has had a positive impact on the spread of infection, slowing its rate of development (Fig. 7)

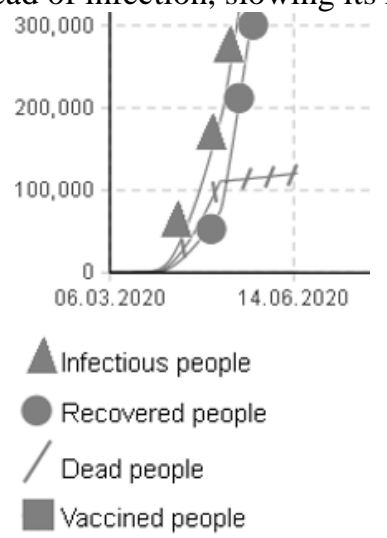

Fig. 7. Event occurrence: "Introducing restrictive measures" in the United States.

3. In the absence of a vaccination process in the United States, the number of cases significantly exceeds the number of cases in the presence of vaccination by almost four times (190 million patients versus 50 million for the same time period of the study -2 years) (Fig. 8)



Fig. 8. Model of the spread of Covid-19 coronavirus infection in the United States of America. Time range 2 years from the beginning of infection without vaccination.

4. The fight against infection is projected to end by 2026, if the current rate of vaccination and the quality of medicine increase are maintained.

\section{Discussion of the results}

1. When comparing the time graphs obtained during the simulation and the real data on the incidence, it can be concluded that they are almost identical, which indicates the correctness of the model and the possibility of using it to predict the further development of infection 
and to draw up a plan for further actions to combat coronavirus on the basis of the obtained forecast.

2. Based on the graphs, it can be concluded that vaccination significantly reduces the rate of infection of people. Thus, it has a positive effect on the number of people susceptible to infection.

3. In the absence of vaccination, the rate of infection continues to increase, thus the number of infected people will constantly increase.

4. Subject to restrictive measures to control the spread of infection, the disease significantly slows down the rate of development.

5. Timely deterrent measures introduced by administrative resources have significantly reduced the rate of infection and the number of deaths, which is clearly observed in the analysis of the graph shown in Figure 7.

6. When comparing the timing of the predicted final victory over coronavirus infection in the Kaluga region and in the United States of America, there is a noticeable difference in two years, which is explained by the higher vaccination rates (at the time of writing, the number of vaccinations against Covid-19 in the United States exceeds the number of vaccinations in Russia by more than 13 times).

7. The quality of health care in terms of infection control increases every month: separate rooms are being built, and the number of places for patients is increasing. This has a positive effect on the recovery of infected people, thereby causing an increase in the number of people who have developed antibodies to the virus. Consequently, the rate of development of the disease slows down with each month to a greater or lesser extent

8. The amplitude of the rate of spread of Covid-19 within the boundaries of different areas have a noticeable dissimilarity, which is associated with a different level of contact between people, as well as the vaccination of the population. In the Kaluga region, the difference between the situation with the availability of vaccination measures and the situation with their absence is insignificant. However, the same cannot be said about these events in the life of the United States of America: without vaccination, Covid-19 will cover the country's population as quickly as possible and medicine simply will not have time to contain such a wave of people infected with coronavirus infection

9. Based on the result of the forecast of the spread of the virus, it can be concluded that the disease will continue to parasitize the lives of the population for several more years. However, the population will feel relatively safe in about 2 years from the moment of writing the article, which is explained by the development of medicine, as well as vaccination of the population. But it is not necessary to stop restrictive measures ahead of time, as this may lead to a new, less controlled and predictable outbreak of the disease. The development of the virus is difficult to predict, because it constantly adapts to a new environment.

\section{Conclusion}

This model is only relevant if the pandemic continues at the same pace. There are many factors that can deviate from the presented model in any direction: early release from restrictive measures, virus mutations, forced vaccination of the population, and other measures that directly affect the main indicators of infection development (ContactRateInfectious and Infection).

In the course of modeling the spread of Covid-19 coronavirus infection in the Kaluga region and the United States of America, a time graph was obtained that reflects the rate of development of the pandemic. The timeline includes four key parameters, namely Infectious people, Recovered people, Dead people, Vaccinated people. 
When considering different borders, it can be concluded that the coronavirus infection shows the maximum amplitude of changes within the country. The spread of the disease occurs mainly in public areas (for example, bus stops, shopping centers) and through contact between family members, as well as through close interaction, for example, between neighbors in the stairwell. Infection occurs by airborne and household contact methods, but infection rates are approximately equal among different age groups.

The spread of the disease directly depends on the measures taken by medicine. Successful response to the pandemic:

1. During the period of the greatest spread of viruses that cause Covid-19, due to the lack of specialized methods of medical treatment, maintenance therapy was used. Quarantine measures in relation to the infected and hypothetically infected have shown their effectiveness.

2. The level of collaboration of research and business activities for medical purposes related to the development of vaccinations and equipment for the diagnosis of patients has increased between higher educational institutions and hospitals, whose task is to effectively process existing and incoming information about the distinctive features and specifics of the virus and its associated diseases.

With the vaccination of the population, the victory of humanity in the fight against Covid-19 will end much faster and more successfully than if the population is not vaccinated against the virus. If the population refuses the vaccine, the fight against infection will continue for a long time, during which time the virus will be able to mutate and bring enough problems and negative consequences for human activity.

It is possible that the technology of risk research, based on the analysis of interactions between people, will create conditions for many states that are favorable for research and the development of a unified action plan for the prevention of further transmission of the virus. Thanks to the infection prediction system, it is possible to identify weak aspects of the population's activities against the virus and in the future make the right decisions to achieve complete victory in this fight, as well as to minimize the rate of disease development and the likelihood of a new outbreak of coronavirus infection.

\section{References}

1. R. Maclntyre, Q. Wang, The Lancet Infectious Diseases (2020)

2. D.K. Nekrasov, M.A. Kuznetsova, K.D. Andreeva, FORCIPE, S (2020)

3. T.N. Bilichenko, Academy of Medicine and Sports, 2 (2020)

4. R. Verity, L.C. Okell, I. Dorigatti, P. Winskill, C. Whit-taker, N. Imai, The Lancet Infectious Diseases (2020)

5. G.G. Onishchenko, T.E. Sizikova, V.N. Lebedev, S.V. Borisevich, BIOpreparaty. Prevention, diagnosis, and treatment, 4 (2020)

6. Resolution of the Government of the Kaluga Region of March 17 (2020)

7. N.G. Kondrashova, Audit and financial analysis, 4, 113 (2020)

8. A.L. Tkachenko, Information technologies in economics, business and management. Materials of the V International Scientific and Practical Conference, 194 (2018)

9. V. Boev, Journal of Applied Informatics, 6 (30), 69 (2010)

10. A.V. Blagov, Modern Applied Science, 9(4), 254 (2015)

11. S.C. Parks, M. Garifullin, R. Dronzek, Proceedings - Winter Simulation Conference, $1038(2005)$

12. A.S. Akopov, L.A. Beklaryan, Knowledge-Based Systems, 174, 103 (2019)

13. A.M. Rudenko, V.I. Rodionova, V.N. Stepanova, Advances in Intelligent Systems and Computing, 726, 1144 (2019)

14. V.S. Stepanov, Health Risk Analysis, 4, 12 (2020) 
15. S. Marino, D.E. Kirschner, J. Theor. Biol., 227(4), 463 (2004)

16. A.G. Bareysha, International Scientific Research Magazine, 1-4(20), 153 (2014)

17. F.M.G. Magpantay, N. Kosovalić, J. Wu, SIAM Journal on Numerical Analysis, 52(2), 735 (2014)

18. A. Godio, F. Pace, A. Vergnano, Int. J. Environ. Res. \& Public Health, 17(10), 3535 (2020) 